This item was submitted to Loughborough's Research Repository by the author.

Items in Figshare are protected by copyright, with all rights reserved, unless otherwise indicated.

\title{
Transient analysis of tyre friction generation using a brush model with
} interconnected viscoelastic bristles

PLEASE CITE THE PUBLISHED VERSION

\section{PUBLISHER}

Professional Engineering Publishing / @ IMECHE

\section{VERSION}

VoR (Version of Record)

\section{LICENCE}

CC BY-NC-ND 4.0

\section{REPOSITORY RECORD}

Mavros, George, Homer Rahnejat, and P.D. King. 2009. "Transient Analysis of Tyre Friction Generation Using a Brush Model with Interconnected Viscoelastic Bristles”. figshare. https://hdl.handle.net/2134/4822. 
This item was submitted to Loughborough's Institutional Repository (https://dspace.lboro.ac.uk/) by the author and is made available under the following Creative Commons Licence conditions.

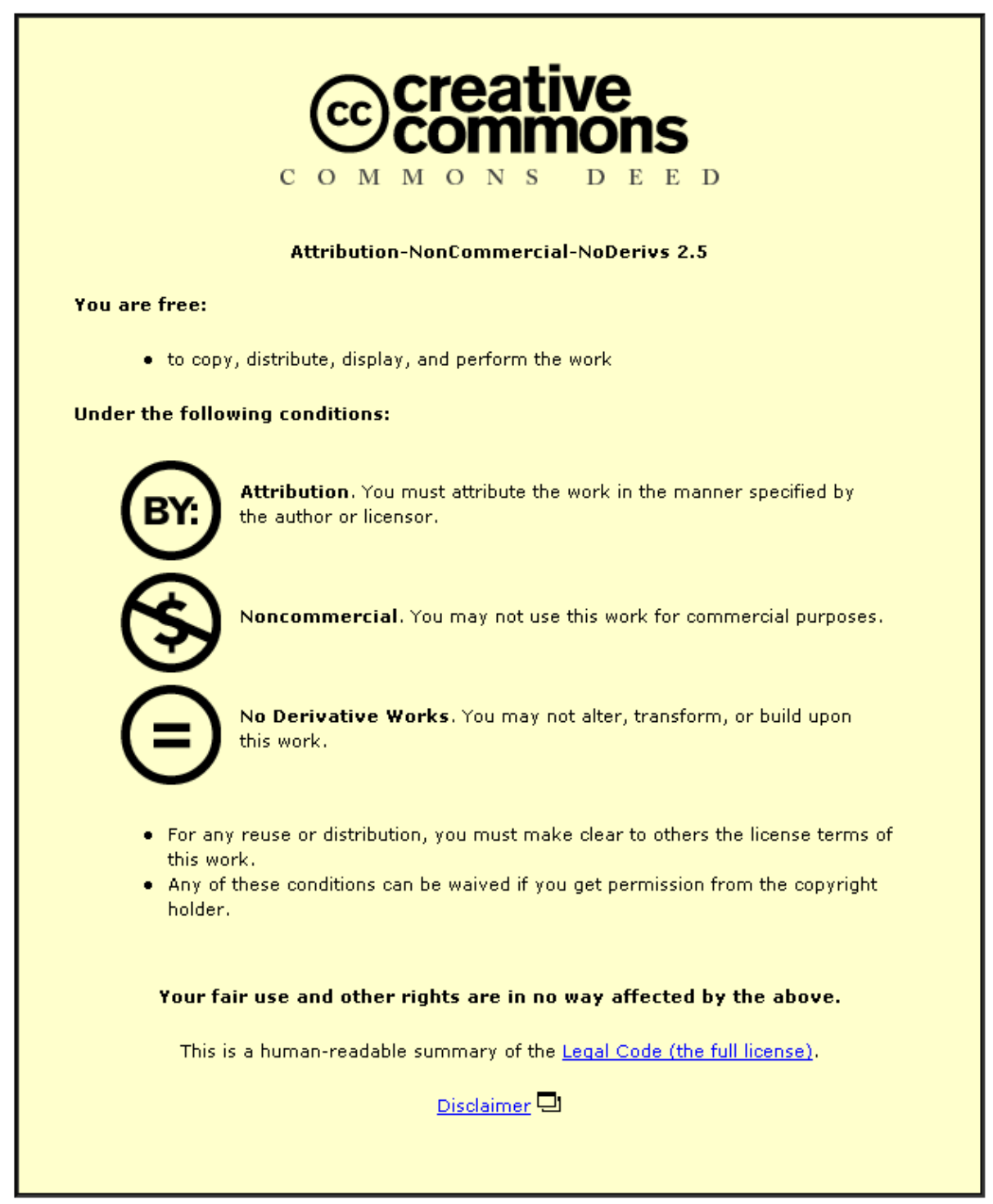

For the full text of this licence, please go to: http://creativecommons.org/licenses/by-nc-nd/2.5/ 


\title{
Transient analysis of tyre friction generation using a brush model with interconnected viscoelastic bristles
}

\author{
G Mavros, H Rahnejat*, and P D King \\ Wolfson School of Mechanical and Manufacturing Engineering, Loughborough University, Loughborough, UK
}

The manuscript was received on 2 February 2004 and was accepted after revision for publication on 11 October 2004.

DOI: $10.1243 / 146441905 X 9908$

\begin{abstract}
An analysis of the mechanism of tyre contact force generation under transient conditions is presented. For this purpose, two different versions of a brush model are used, both with inertial and viscoelastic properties. The first model consists of independent bristles, while the second, with a more realistic scenario, introduces viscoelastic circumferential connections between the sequential bristles, which affect the lateral degrees of freedom. Friction between the tyre and the ground follows an experimentally verified stick-slip law. For the model with independent bristles, the state of each bristle at any instant of time depends only on the state of the same bristle at a previous time step. In the second model, the instantaneous state depends on the state of the same bristle at the preceding time step, as well as on the state of the two adjacent bristles at the same time. Simulation results reveal the differences between the two models and most importantly show how transient friction force generation may differ substantially from steady state predictions. The findings suggest that transient tyre behaviour should not be attributed solely to the contributions of the flexible belt and carcass. On the contrary, the observed transience in the neighbourhood of the contact patch should also be taken into account.
\end{abstract}

Keywords: tyre, transient dynamics, stick-slip friction, viscoelasticity, lateral tyre force, self-aligning moment, tyre modelling

\section{INTRODUCTION}

Steady state tyre models provide a means of predicting forces under steady state operating conditions and can be divided into two main categories, namely empirical and physical models [1]. Empirical models are based on the construction of force versus slip functions that successfully fit a series of experimental measurements. The most widely used empirical model is the magic formula initially conceived by Bakker and Pacejka [2]. On the other hand, physical models are concerned with the mechanism of tyre force generation and use mathematical tyre representations, which can be solved analytically or, in more elaborate cases, via computer simulations. Such models are proposed, for example, in the detailed analyses carried out by Sakai [3-6],

*Corresponding author: Wolfson School of Mechanical and Manufacturing Engineering, University of Loughborough, Loughborough, Leicestershire LE11 3TU, UK.
Gim and Nikravesh [7-9], and Bernard et al. [10]. Hitherto, empirical models and especially the magic formula seem to have yielded more realistic results for an extended combination of cases. This could be due to the difficulties in modelling the highly complex structure of the tyre and its interaction with the road. As a result, researchers have tended to use steady state empirical models for the majority of cases, even for the simulation of transient handling conditions.

Nevertheless, transient conditions introduce phenomena that cannot be neglected, the most common being a phase lag between the actual steering command and the build-up of tyre forces. This is caused mainly by the flexibility of the tyre carcass and belt and the interaction of the tyre inertia with that of the vehicle. These factors may also introduce vibrations, acting in general as a filter between the contact patch and the wheel hub. These phenomena can be compensated for by introducing a delay between the tyre kinematic conditions that produce 
a specific force and the actual application of this force on the wheel hub. A more elaborate solution is the modelling of the tyre carcass and/or belt as a separate dynamic system and its combination with a steady state force generation procedure, as demonstrated by the transient version of the magic formula tyre model [11]. Finally, the relaxation length concept can be employed in order to describe simple cases of transient force transfer from the tread to the wheel hub under transient cornering, whereas Pacejka [12] and Zegelaar et al. [13] deal with more complicated transient problems such as the response of a tyre to lateral vibrations and braking torque variations respectively.

The aforementioned approaches deal mainly with the transient conditions involved in the transfer of tyre forces to the wheel hub. Another condition, which is often neglected, concerns the transient build-up of tyre forces on the contact patch itself. Little is known about how friction forces between the rubber and the ground are generated and how tyre characteristics can affect the generation of these forces under rapidly changing operating conditions. It is expected that the frictional properties of the contact area would interact with the dynamic properties of the tyre, such as its viscoelasticity and inertia, and affect the transient generation of contact forces. While the instantaneous response of rubber yields its elastic properties, the finite nature of an impact (i.e. impact time) leads to viscoelastic relaxation, which tends to reduce the contact pressure distribution and spread the area of contact. These effects have been reported by Johnson [14], Jacobson and Kalker [15], and Naghieh et al. [16] and take place rapidly through the relaxation time of the viscoelastic material, typically of the order of hundredths to tenths of a second. It should be noted that the carcass would behave instantaneously as elastic at the leading edge of the contact, and relax viscoelastically while in the contact domain.

Many attempts have been made to simulate transient tyre behaviour using variations of bristlebased models. Van Zanten et al. [17] have used a brush model for the generation of contact forces. The bristles are massless and undamped and the forces can be calculated if the kinematic conditions of the tread are known. These conditions in turn are calculated by simulation of a separate dynamic model of the carcass. Clearly, it is a case of a kinematic contact force model working in conjunction with a dynamic rim-carcass-belt model. Zhou et al. [18] have used a modified transient version of the multispoke model presented in reference [19]. Again, the spokes are massless and undamped and a rigid spoke base with lateral and longitudinal degrees of freedom is incorporated to account for the carcass behaviour. The contact force generation is in fact transient, as it involves updating the states of all spokes under consideration to $t+\mathrm{d} t$ from the corresponding states at $t$. Furthermore, the motion of the rigid bristle base is calculated dynamically. Nevertheless, the contact problem is solved in a purely kinematic manner, and the bristle tip friction neglects inertial and damping properties of the tread. A more consistent approach should look at the effects of these properties on the contact force generation in unison.

In the present analysis, a brush model with distributed inertial, elastic, and damping properties is used to simulate the transient contact force generation. The analysis is taken one step further by connecting the bristles together, using springs and dampers, representing a Kelvin element. This approach is thought to be most appropriate for a transient contact model. It is also in line with the approach used in contact mechanical analysis of viscoelastic solids by Jacobson and Kalker [15] and Naghieh et al. [16], where the viscoelastic material is considered as a semi-infinite half-space in contact with a rigid surface. Otherwise, when the bristles are allowed to move independently, it is possible to observe large differences in the velocity of adjacent bristles, as a result of rapidly changing kinematic and frictional conditions. In reality, the motion of each bristle is restrained by the motion of the adjacent bristles, which is achieved by connecting the bristles to each other.

\section{DESCRIPTION OF THE MODEL}

The model used as a starting point for the analysis is the simple version of the steady state brush model described in reference [20]. There is evidence [21] that the length of the contact patch does not change significantly with an increase in the forward velocity. Furthermore, if a constant forward velocity is considered, the use of a model with a constant contact patch length and parabolic pressure distribution can serve as a good starting point for the analysis [14].

A brief description of the steady state version of the tyre model is given below, before proceeding with the necessary modifications in order to facilitate the simulation of transient tyre behaviour.

The tyre tread is modelled as a one-dimensional series of bristles distributed on the tyre periphery, as shown in Fig. 1. The bristles incorporate anisotropic stiffness and damping in the lateral and longitudinal directions, and the distributed tread mass on the tyre periphery is also taken into account by attaching an infinitesimal mass to the end of each bristle. As mentioned, the pressure distribution is 


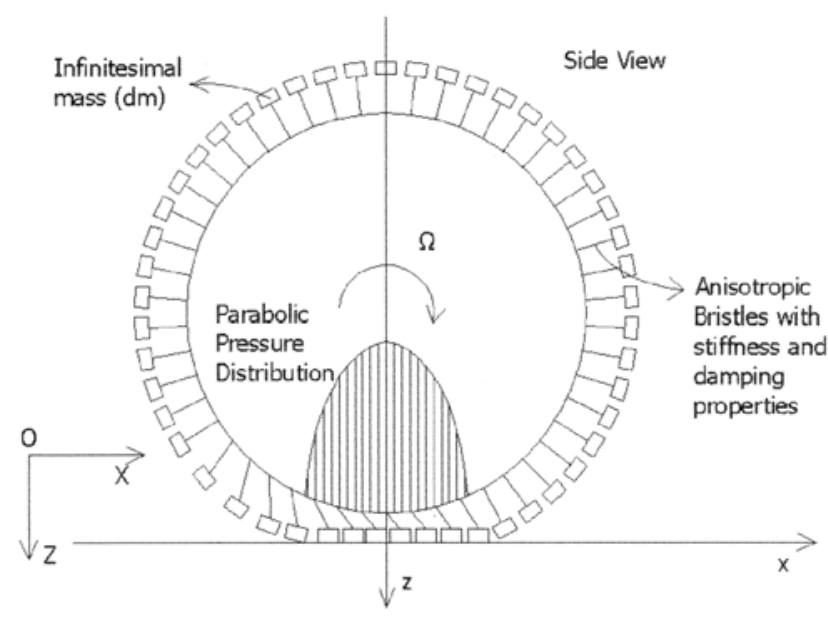

Fig. 1 Side elevation view of the basic bristle model

assumed to be parabolic and the length of the contact patch remains constant.

All tyre properties are written per unit length of the contact patch. The mass distribution along the tyre tread is $C=\mathrm{d} m / \mathrm{d} x$, the stiffness coefficients per unit tread length are $K_{x x}=(\mathrm{d} F / \mathrm{d} x) / \mathrm{d} x$ and $K_{y x}=(\mathrm{d} F / \mathrm{d} y) / \mathrm{d} x$ for the longitudinal and lateral deformations of the bristles respectively, and the damping coefficients are $D_{x x}=\left(\mathrm{d} F / \mathrm{d} u_{x}\right) / \mathrm{d} x$ and $D_{y x}=\left(\mathrm{d} F / \mathrm{d} u_{y}\right) / \mathrm{d} x$, where $u_{x}$ and $u_{y}$ denote the rates of change in the longitudinal and lateral deformations of the bristles respectively.

Figure 1 shows a side view of the tyre model, including the normal pressure distribution, while Fig. 2 shows a top view of the model, depicting an arbitrary position of the infinitesimal mass $\mathrm{d} m$, which corresponds to a length $\mathrm{d} x$ of the tyre tread (i.e. $\mathrm{d} m=C \mathrm{~d} x$ ). The bristle, connecting the mass to the wheel periphery, is deformed laterally, as well as longitudinally, and the mass may or may

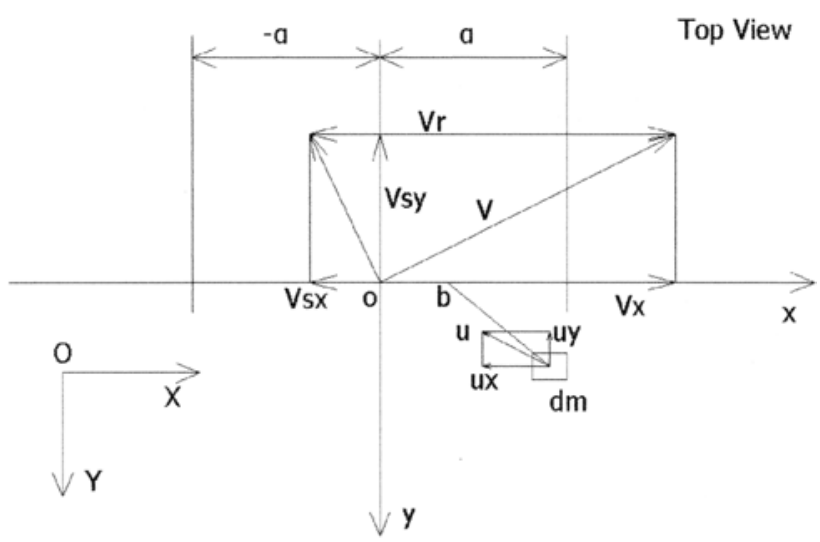

Fig. 2 Top plan view of the basic bristle model not be sliding on the ground, depending on the viscoelastic restoring forces applied by the bristle, the normal force at the specific position, and the coefficient of friction.

The normal force distribution throughout the length of the contact patch is given by the parabolic equation

$$
F_{\text {vertical }}=\frac{3 F_{z}}{4 \alpha}\left(1-\frac{x}{a}\right)^{2}
$$

where $F_{z}$ denotes the total vertical force applied on the wheel hub and $2 a$ is the total length of the contact patch.

Two different sets of axes are used in order to describe the motion of the tyre and its components. The global frame of reference $(O X Y Z)$ is attached to the ground, while a second frame of reference (oxyz) has its origin on the point in the contact patch where the vertical line from the centre of the wheel plane meets the ground. Both frames are shown in Figs 1 and 2.

Point $b$, where the bristle is connected to the tyre periphery (i.e. the bristle base), enters the contact patch at coordinates $(x, y, z)=(\alpha, 0,0)$ on the moving frame of reference and travels throughout the contact patch with velocity $V_{\mathrm{d}}=\omega R_{\mathrm{d}}$, where $R_{\mathrm{d}}$ is the radius of the vertically loaded tyre under pure rolling condition. The longitudinal component of the velocity of the of the bristle base relative to the ground (global frame of reference) is $V_{\mathrm{s} x}$, the lateral component being $V_{s y}$. When the vertical force results in the generation of a high enough frictional force, the infinitesimal mass $\mathrm{d} m$ sticks on the ground. In any other case, the mass moves with respect to the ground with a sliding velocity $u$, consisting of $u_{x}$ and $u_{y}$ in the longitudinal and lateral directions respectively.

Irrespective of whether the tyre is slipping or not, the velocity of travel of point $b$ throughout the length of the contact patch is $V_{\mathrm{d}}$. Obviously, this is the velocity throughout the normal force distribution as well. Thus, the vertical force on point $b$ varies according to the following relationships

$$
\begin{aligned}
F_{\text {vertical }} & =\frac{3 F_{z}}{4 \alpha}\left(1-\frac{x_{\mathrm{d}}}{a}\right)^{2} \mathrm{~d} x \\
\dot{x}_{\mathrm{d}} & =V_{\mathrm{d}}
\end{aligned}
$$

Friction between the tread and the road follows a simplified stick-slip law derived from experimental measurements by Braghin et al. [22]. 
The simplified friction law is summarized by the relations

$$
\begin{aligned}
& f=-F_{\text {external }} \quad \text { for } u=0 \quad \text { AND } \\
& F_{\text {external }} \leq F_{\text {max }}=\text { factor } \cdot \mu_{\mathrm{k}} \cdot F_{\text {vertical }}=\mu F_{\text {vertical }} \\
& f=\mu_{\mathrm{k}} F_{\text {vertical }} \quad \text { for }|u|>0 \quad \text { OR } \\
& \left|F_{\text {external }}\right|>\mu F_{\text {vertical }}
\end{aligned}
$$

where $F_{\text {external }}$ denotes the forces applied on the mass by the bristle, $u$ denotes the sliding velocity of the mass with respect to the ground, $\mu_{\mathrm{k}}$ is the coefficient of kinetic friction, $F_{\text {vertical }}$ is the vertical force corresponding to the longitudinal position of the mass, and $F_{\max }$ is the maximum friction force, which can be obtained by multiplying the kinetic coefficient friction $\mu_{\mathrm{k}} F_{\text {vertical }}$ by a factor of $>1$ such that static friction $\mu$ is given by the relation $\mu=$ factor $\mu_{\mathrm{k}}$. During simulation, the stick-slip conditions described by relations (4) and (5) are checked using a velocity transition threshold. For velocities below the value of the threshold, the infinitesimal mass is considered to be stationary, while sliding occurs for velocities greater than the threshold, as proposed by Karnopp [23].

The value of friction $\boldsymbol{f}=f_{x}+f_{y}$ is determined by equations (4) and (5). If the sticking condition is satisfied, then the magnitude of the friction force equates to the magnitude of the forces applied by the bristle. When the mass is sliding on the ground, the magnitude of the friction force becomes

$$
|\boldsymbol{f}|=\mu_{\mathrm{k}} \frac{3 F_{z}}{4 \alpha}\left(1-\frac{x_{d}}{a}\right)^{2} \mathrm{~d} x
$$

The components of $\boldsymbol{f}$ in the longitudinal and lateral directions are

$$
\begin{aligned}
& f_{x}=\frac{u_{x}}{\sqrt{u_{x}^{2}+u_{y}^{2}}} \mu_{\mathrm{k}} \frac{3 F_{z}}{4 \alpha}\left(1-\frac{x_{\mathrm{d}}}{a}\right)^{2} \mathrm{~d} x \\
& f_{y}=\frac{u_{y}}{\sqrt{u_{x}^{2}+u_{y}^{2}}} \mu_{\mathrm{k}} \frac{3 F_{z}}{4 \alpha}\left(1-\frac{x_{\mathrm{d}}}{a}\right)^{2} \mathrm{~d} x
\end{aligned}
$$

The differential equations, describing the motion of the mass in the longitudinal and lateral directions are written as follows (in accordance with Fig. 2)

$$
\begin{aligned}
& \dot{u}_{x} C \mathrm{~d} x=\left(x_{\mathrm{s}}-x\right) K_{x} \mathrm{~d} x+\left(V_{\mathrm{s} x}-u_{x}\right) D_{x} \mathrm{~d} x-f_{x} \\
& \dot{u}_{y} C \mathrm{~d} x=\left(y_{\mathrm{s}}-y\right) K_{y} \mathrm{~d} x+\left(V_{\mathrm{s} y}-u_{y}\right) D_{y} \mathrm{~d} x-f_{y} \\
& \dot{x}_{\mathrm{s}}=V_{\mathrm{s} x} \\
& \dot{y}_{\mathrm{s}}=V_{\mathrm{s} y}
\end{aligned}
$$

In the steady state model [20], the motion of the infinitesimal mass is followed throughout the contact patch and is representative of the motion of all such elements in contact with the ground. Furthermore, the steady state model is run for pure braking, traction, cornering, or combinations of the above, as shown in the general case represented by Fig. 2. The transient model is run for purely cornering conditions, so that $V_{\mathrm{s} x}$ vanishes, and equations (9) and (11) yield zero displacements and velocities in the longitudinal direction.

While $V_{\text {sy }}$ is constant in the steady state model, it changes in each time step in the transient model. Consequently, the state of all infinitesimal masses in contact with the ground can no longer be obtained by following one representative mass throughout the contact patch. Instead, the state of a mass $\mathrm{d} m$ at $t+\mathrm{d} t$ results from the state of the same mass at $t$.

In order to solve the problem, the vectors of the state variables, positions, and velocities of all infinitesimal masses forming the contact patch have to be defined. If the length of the contact patch is $2 a=n \mathrm{~d} x$, then $n$ infinitesimal masses are involved in the problem. At a random operating point, for example at time $t$, each mass is characterized by its velocity and position in the oxy plane. At time $t+\mathrm{d} t$, every mass has moved one place towards the end of the contact patch, travelling a distance of $\mathrm{d} x=V_{\mathrm{d}} \mathrm{d} t$. This sequential switching is also reflected in the state vectors, so that the state of the $i+1$ th mass at time $t+\mathrm{d} t$ can be calculated by using the state of the $i$ th mass at time $t$. In order for each mass exactly to take the place of the one adjacent to it, the time step has to be constant and the number of masses has to be set according to the relationship

$$
n=\frac{2 a}{V_{\mathrm{d}} \mathrm{d} t}
$$

with $V_{\mathrm{d}}$ being fixed as well.

Thus, the total number of infinitesimal masses (corresponding to infinitesimal lengths $\mathrm{d} x$ ) depends on the time step size and the circumferential velocity, $V_{\mathrm{d}}$. Considering numerical stability issues, the size of the time step should be the dominating factor and has to be set $a$ priori. Nevertheless, for a given length $2 a$ and velocity $V_{\mathrm{d}}$, a numerically effective time step could result in the generation of a small number of masses in contact with the ground. For example, if $V_{\mathrm{d}}=40 \mathrm{~m} / \mathrm{s}, 2 a=0.16 \mathrm{~m}$, and $\mathrm{d} t=0.0001 \mathrm{~s}$, then equation (13) yields $n=40$, which means that the contact patch has been divided into 40 discrete parts with mass $\mathrm{d} m$ and length $\mathrm{d} x$. Obviously, 200 or 300 segments would give a smoother and more accurate force generation mechanism. The dependence of the number of elements 


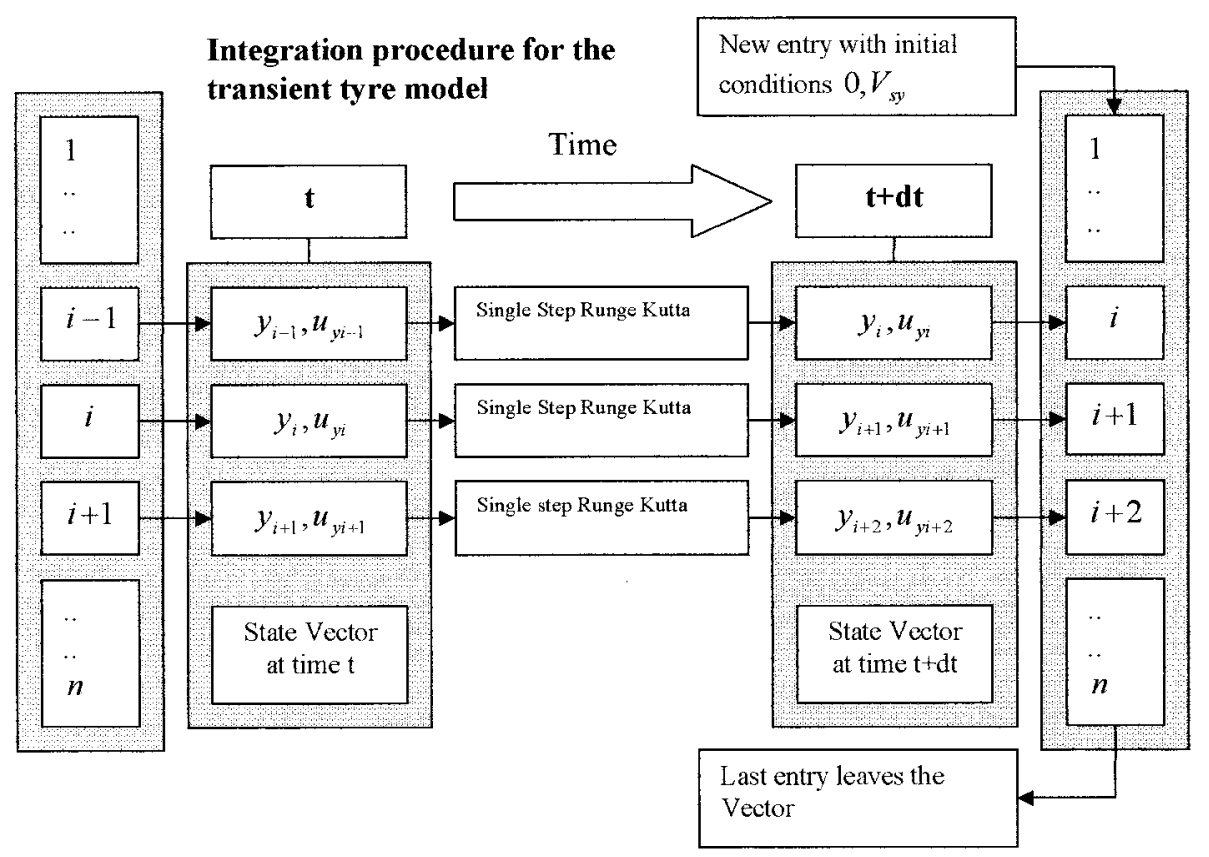

Fig. 3 Time marching in transient analysis

on the rolling velocity and the fact that the latter needs to remain constant are the main disadvantages of the proposed formulation and currently limit the potential uses of the model.

The fourth-order fixed-step Runge-Kutta method is used for advancing the state of each infinitesimal mass from $t$ to $t+\mathrm{d} t$. The graphical representation in Fig. 3 shows the integration procedure according to the previous analysis.

The $n$ infinitesimal masses on the left are linked to their states at time $t$. One step of the integration algorithm is applied on every single vector entry, using as initial conditions the positions and velocities included in the state vector at time $t$. In this manner, a single step of integration is applied for $n$ different initial conditions, as opposed to the steady state model, where, starting from one set of initial conditions, the integration is applied for $n$ sequential steps, following a single mass $\mathrm{d} m$, from the beginning to the end of the contact area. Meanwhile, every mass $\mathrm{d} m$ moves for a distance of $\mathrm{d} x=V_{\mathrm{d}} \mathrm{d} t$, so that the $i$ th entry replaces the $i+1$ th entry, and so forth. Thus, the updated states obtained by the integrator are moved one place towards the end of the state vector, in such a way that an empty space is created in the beginning of the vector, whereas at the same time the $n$th entry is 'pushed' out of the vector. The empty space created in the beginning is then occupied by a new mass with the initial conditions $0, V_{s y}$, where $V_{s y}$ is the new value of the lateral sliding velocity, according to an increase in the slip angle in a transient manner. Owing to the continuous nature of this procedure, the $i$ th entry appears on both sides of the integrator: on the right, it appears as a result of the integration of the $i-1$ th state, while on the left it appears as an initial state for the $i$ th entry.

\section{MODEL WITH INTERCONNECTED BRISTLES}

The interconnect ions between bristles are realized by introducing a Kelvin element, affecting the relative motion of the bristles in the lateral direction. Now, equation (10) becomes

$$
\begin{aligned}
\dot{u}_{y} C \mathrm{~d} x= & \left(y_{\mathrm{s}}-y\right) \cdot K_{y} \mathrm{~d} x+\left(V_{\mathrm{s} y}-u_{y}\right) D_{y} \mathrm{~d} x \\
& -\left(y-y_{i-1}\right) K_{\text {inter }} \mathrm{d} x-\left(y-y_{i+1}\right) K_{\text {inter }} \mathrm{d} x \\
& -\left(u_{y}-u_{y i-1}\right) D_{\text {inter }} \mathrm{d} x \\
& -\left(u_{y}-u_{y i+1}\right) D_{\text {inter }} \mathrm{d} x-f_{y}
\end{aligned}
$$

The integration procedure is similar to that described previously, only, this time, equation (14) uses the displacements and velocities of the adjacent bristles at time $t$ to calculate the state of the $i$ th bristles at time $t+\mathrm{d} t$. This is shown graphically in Fig. 4. At state 0 , the bristles are characterized by zero initial conditions. Velocity $V_{\mathrm{s} y}$ is applied, so the first application of the Runge-Kutta algorithm on every bristle results in state 1 , as shown in the diagram. During this first application of the integrator, the differences $\left(y-y_{i \pm 1}\right)$ and $\left(u_{y}-u_{y i \pm 1}\right)$ in equation (14) are all equal to zero. On the otherhand, state 1 shows differences in the lateral displacements and velocities of adjacent bristles, as a result of different frictional 


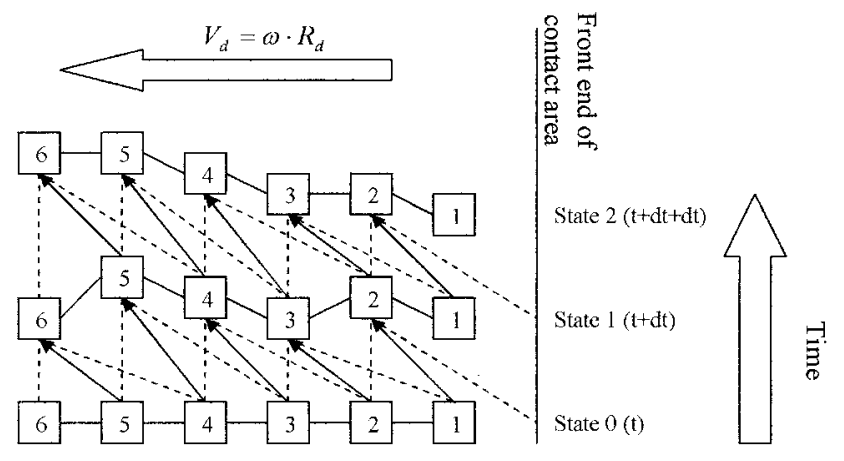

Fig. 4 Stepwise integration process

conditions during the first integration step. These differences are taken into account by equation (14), when the integrator advances from state 1 to state 2, and so on.

\section{SIMULATION RESULTS - DISCUSSION}

Before proceeding with the transient simulations, the structural parameters of the tyre model need to be identified. This is achieved by comparing the steady state version of the model with a generic passenger tyre as described by the magic formula. Parameters such as the lateral and longitudinal stiffness coefficients and the coefficient of kinetic friction may be determined by this procedure. Internal damping of the tyre rubber does not affect significantly the shape of the steady state diagrams and thus cannot be obtained by them. Instead, the damping coefficient of the Kelvin elements can be estimated using rubber blocks consisting of the same rubber compound as the tyre tread.

In addition, some issues related to the simulation of stick-slip friction need to be addressed. While the kinetic friction coefficient can be derived by the saturated area of steady state diagrams, such as those obtained by the magic formula, the coefficient of static friction and the velocity threshold for the transition between stiction and sliding need to be determined through experimental measurements. For this purpose, the measurements described in reference [22] were simulated. In brief, a rubber block represented by a mass connected to a Kelvin element is held steadily against a moving platform. Experimental results [22] show that the existence, amplitude, and frequency of the stick-slip motion of the rubber block depend largely on the platform velocity and the normal force applied on the specimen. In turn, simulation of the actual measurements shows that, given the above parameters, the transition threshold depends largely on the time step and method of integration. For the purpose of the present analysis, a fourth-order fixed-step RungeKutta method was used. It was found that, although a wide range of time steps provides numerically stable results, for each of these different time steps a specific transition threshold exists that depicts the stick-slip behaviour of the rubber accurately. Taking into account the considerations presented above, the parameters in Table 1 were used throughout the transient simulations.

The transient response of a tyre in a lateral manoeuvre is chosen as the case study for this paper. The wheel is moving forward with a constant velocity of $10 \mathrm{~m} / \mathrm{s}$, while an increasing lateral velocity is imposed on the wheel rim. The result is a transient increase in lateral slip at a constant rate of $30.96 \mathrm{deg} / \mathrm{s}$. Practically, this manoeuvre is approximately equivalent to the transient increase in slip angle of the rear tyres of a car as a result of oversteering behaviour, when the driver ceases upon acceleration mid-way through a tight corner. The specific test case can be regarded as a virtual transient test using a typical drum or flat-belt machine and is preferred, because it enforces tyre operation well beyond the limits of adhesion.

In Fig. 5, the dotted line corresponds to the results obtained by the steady state version of the model with non-interconnected bristles, while the solid line represents the behaviour of the transient version of the same model. Both models yield similar results in the low, linear range of the force-slip diagram. As the slip ratio increases and the graphs enter the non-linear region of operation, microscopic stickslip action between the tread elements and the road leads to minor fluctuations, captured by the transient model. With a further increase in the slip ratio, higher amplitudes of oscillation are predicted by the transient model. These oscillations can reach up to 10 per cent of the total lateral force developed by the tyre. As the slip ratio increases further, the period and amplitude of oscillations decrease continually, and finally the response smoothens completely at the saturated area of operation. It is suspected that

Table 1 Simulation parameters

\begin{tabular}{|c|c|c|c|c|c|c|c|c|c|}
\hline $\begin{array}{l}l \\
(\mathrm{~m})\end{array}$ & $\begin{array}{l}R \\
(\mathrm{~m})\end{array}$ & $\begin{array}{l}b \\
(\mathrm{~m})\end{array}$ & $\begin{array}{l}F_{z} \\
(\mathrm{~N})\end{array}$ & $\begin{array}{l}K_{y} \\
\left(\mathrm{~N} / \mathrm{m}^{2}\right)\end{array}$ & $\begin{array}{l}K_{\text {inter }} \\
\left(\mathrm{N} / \mathrm{m}^{2}\right)\end{array}$ & $\begin{array}{l}D_{y} \\
\left(\mathrm{~N} \mathrm{~s} / \mathrm{m}^{2}\right)\end{array}$ & $\begin{array}{l}D_{\text {inter }} \\
\left(\mathrm{N} \mathrm{s} / \mathrm{m}^{2}\right)\end{array}$ & $\mu_{\mathrm{k}}$ & $\mu$ \\
\hline 0.065 & 0.29 & 0.18 & 4150 & $9 \times 10^{6}$ & $3.6 \times 10^{6}$ & $8 \times 10^{2}$ & $3.2 \times 10^{2}$ & 0.9 & 1.17 \\
\hline
\end{tabular}

Time step $=1 \times 10^{-4} \mathrm{~s}$, velocity transition threshold $=0.012 \mathrm{~m} / \mathrm{s}$. 


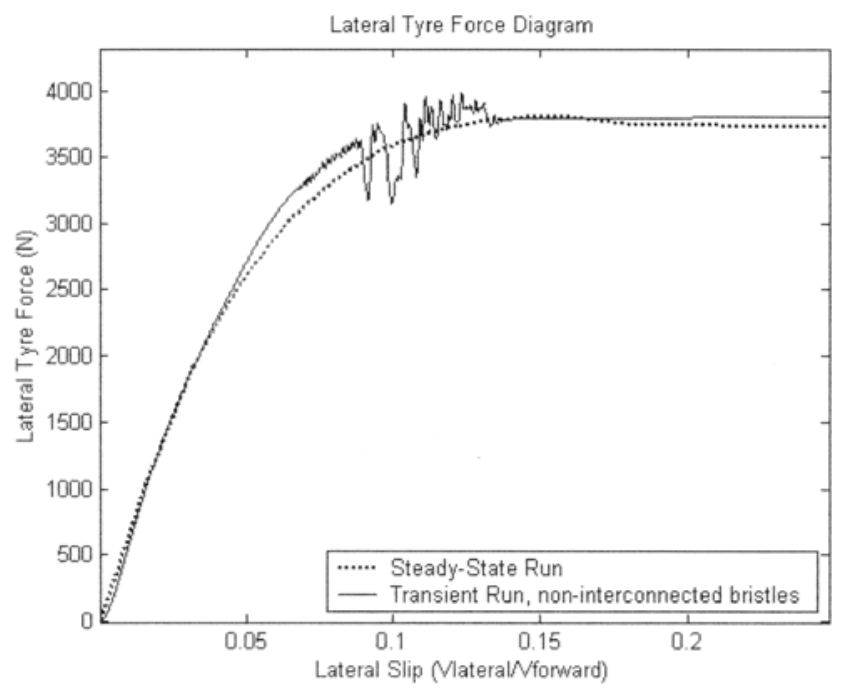

Fig. 5 Lateral tyre behaviour with lateral slip under steady state and transient conditions

the three sequential drops in lateral force predicted by the transient model could alter the response of a vehicle significantly. Experience indicates that, just before reaching saturation, tyres tend to exhibit macroscopic stick-slip behaviour, sensed indirectly by the driver as a discontinuity in the build-up of yaw rate, which could be attributed to the aforementioned drops in the lateral force. The discontinuous tyre marks on the road could also be the result of the aforementioned drops in the lateral force.

Fluctuations in self-aligning moment are not only evident, but also magnified, as indicated in Fig. 6 . While, in the beginning, steady state and transient responses are almost identical, multiplication of the lateral force distribution with the corresponding

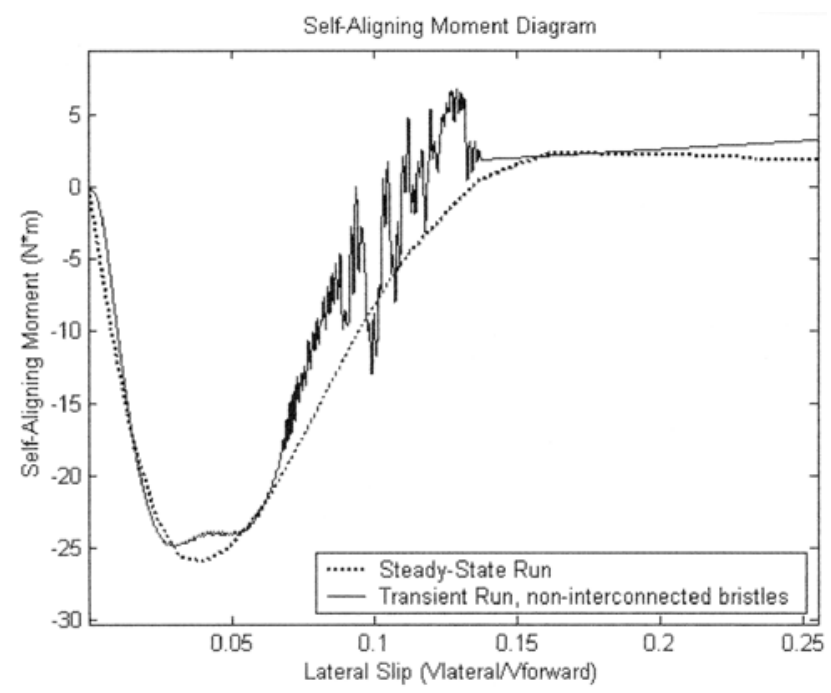

Fig. 6 Self-aligning moment change with lateral slip under steady state and transient conditions

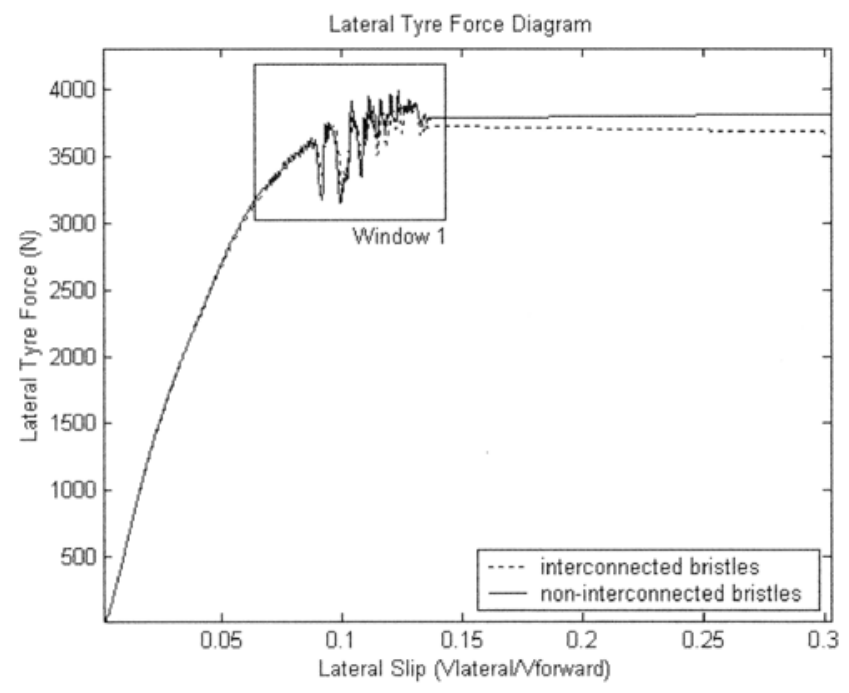

Fig. 7 Lateral forces as predicted by both transient models, one with free and one with interconnected bristles

distances from the vertical axis results in more intense oscillations, which are even more pronounced after the peak value of self-aligning moment in the range of slip ratios between 0.045 and 0.14 . As expected, the transient curve smoothens out towards the end of the graph, inside the region of saturated operation.

Figures 7 to 10 deal with the effect of the interconnections between bristles. Figures 9 and 10 depict two windows from the lateral force and the selfaligning moment variations shown in Figs 7 and 8 respectively. Interconnections clearly reduce the tendency of the bristles to oscillate, especially in

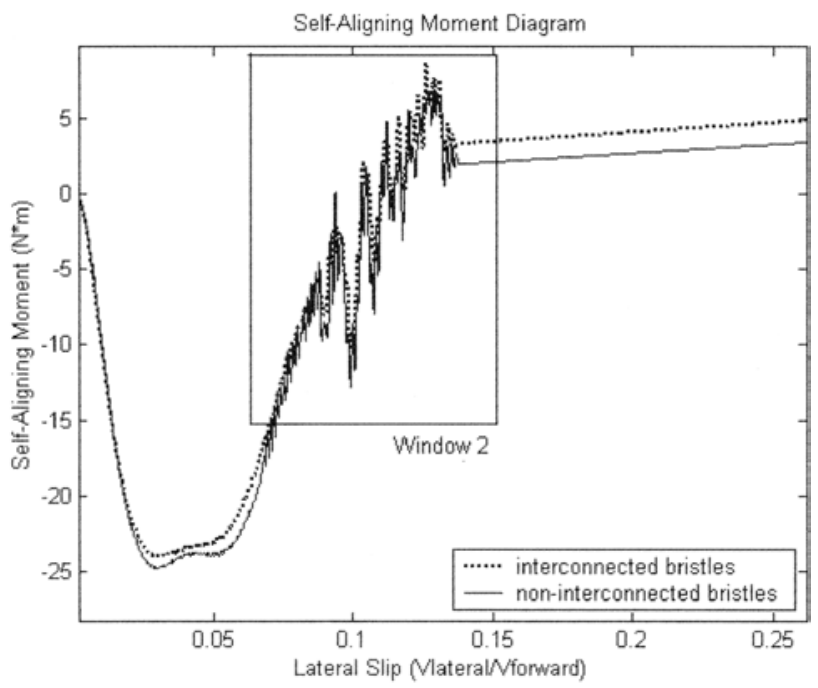

Fig. 8 Self-aligning moment as predicted by both transient models, one with free and one with interconnected bristles 


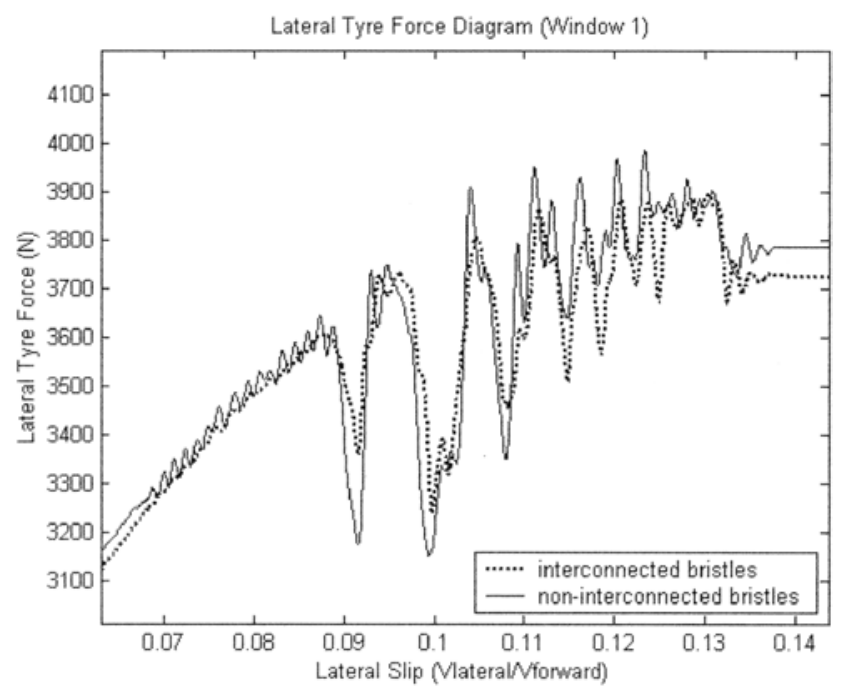

Fig. 9 Magnified window from Fig. 7, showing a comparison between the fluctuations in lateral force, as predicted by the two transient models

the first part of the time history. Nevertheless, it does not seem to affect the severity of the major fluctuations in the lateral force or the self-aligning moment.

Another interesting observation refers to the fact that the model with interconnected bristles generates a slightly lower lateral force in the region of saturated operation. The overall tyre force is calculated as the force applied by the bristles on the wheel rim and not as a friction force applied by the road to the bristles. This approach was preferred,

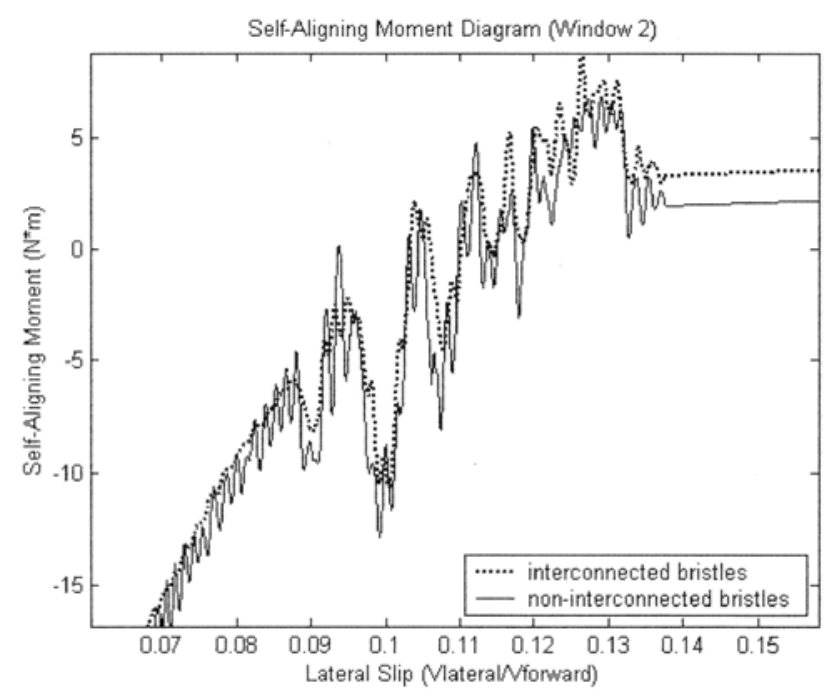

Fig. 10 Magnified window from Fig. 8, showing a comparison between the fluctuations in selfaligning moment, as predicted by the two transient models taking into account the effect of internal damping, which should alter the energy balance between the forces generated along the contact patch and the forces transmitted to the rim. The higher the lateral velocity (saturated region of operation), the more intense is the effect of the inertia of the tyre tread. Because the bristles are not interconnected, there is no wave propagation and energy dissipation along the tyre tread, and the increased forces generated by the bristles are transferred directly to the rim. On the other hand, interconnections between the bristles enable extra energy dissipation and reduce the overall deflection of the bristles. For the sake of computational cost, the number of interconnected bristles is confined to those just covering the contact patch length. In a full model with bristles distributed all around the tyre periphery, wave propagation and energy dissipation would continue outside the contact patch and the tendency for generation of an increasing force with lateral velocity would be completely eliminated. Nevertheless, the results show that, even though a reduced number of bristles is used, the interconnections between the bristles yield sensible results in a reasonable period of time.

\section{CONCLUSIONS}

The present analysis provides strong evidence that the generation of friction force under transient manoeuvres should not be taken for granted. Transient simulations and experimental measurements of transient tyre behaviour have been carried out. Rarely, however, has the aim been detailed investigation of the mechanism of friction force generation. The viscoelastic behaviour of the tyre tread and its most appropriate modelling for the purpose of tyre mechanics should play a more important role in tyre force estimation. Furthermore, a number of highly varying friction laws, depending largely on the viscoelastic properties of rubber, should be used and compared with the experimental findings. Finally, although the model used for the investigations demonstrates certain limitations in terms of practical application, it can be used as a starting platform for the investigation of tyre viscoelastic friction and can provide useful information for the development of more effective tyre models.

\section{REFERENCES}

1 Pacejka, H. B. and Sharp, R. S. Shear force development by pneumatic tyres in steady-state condition: a review of modelling aspects. Veh. Syst. Dynamics, 1991, 20, 121-176. 
2 Bakker, E. and Pacejka, H. B. Tyre modelling for use in vehicle dynamics studies. SAE paper 870421, 1987.

3 Sakai, H. Theoretical and experimental studies on the dynamic properties of tyres. Part 1: review of theories of rubber friction. Int. J. Veh. Des., 1981, 2(1), 78-110.

4 Sakai, H. Theoretical and experimental studies on the dynamic properties of tyres. Part 2: experimental investigation of rubber friction and deformation of a tyre. Int. J. Veh. Des., 1981, 2(2), 182-226.

5 Sakai, H. Theoretical and experimental studies on the dynamic properties of tyres. Part 3: calculation of the six components of force and moment of a tyre. Int. J. Veh. Des., 1981, 2(3), 335-372.

6 Sakai, H. Theoretical and experimental studies on the dynamic properties of tyres. Part 4: investigations of the influences of running conditions by calculation and experiment. Int. J. Veh. Des., 1982, 3(3), 333-375.

7 Gim, G. and Nikravesh, P. E. An analytical model of pneumatic tyres for vehicle denamic simulations. Part 1: pure slips. Int. J. Veh. Des., 1990, 11(6), 589-618.

8 Gim, G. and Nikravesh, P. E. An analytical model of pneumatic tyres for vehicle dynamic simulations. Part 2: comprehensive slips. Int. J. Veh. Des., 1991, 12(1), $19-39$.

9 Gim, G. and Nikravesh, P. E. An analytical model of pneumatic tyres for vehicle dynamic simulations. Part 3: validation against experimental data. Int. J. Veh. Des., 1991, 12(2), 217-228.

10 Bernard, J. E., Segel, L., and Wild, R. E. Tyre shear force generation during combined steering and braking maneuvers. SAE paper 770852, 1977.

11 Pacejka, H. B. and Besselink, I. J. Magic formula tyre with transient properties. Veh. Syst. Dynamics Suppl., 1997, 27, 234-249.

12 Pacejka, H. B. Analysis of the dynamic response of a rolling string-type tire model to lateral wheel-plane vibrations. Veh. Syst. Dynamics, 1972, 1, 37-66.

13 Zegelaar, P. W. A. and Pacejka, H. B. Dynamic tyre responses to brake torque variations. Veh. Syst. Dynamics Suppl., 1997, 27, 65-79.

14 Johnson, K. L. Contact mechanics, 1985 (Cambridge University Press, Cambridge).

15 Jacobson, B. O. and Kalker, J. J. Rolling contact phenomena, 2000 (Springer, Vienna).

16 Naghieh, G. R., Rahnejat, H., and Jin, Z. M. Characteristics of frictionless contact of bonded elastic and viscoelastic layered solids. Wear, 1999, 232, 243-249.

17 Van Zanten, A., Erhardt, R., and Lutz, A. Measurement and simulation of transients in longitudinal and lateral tyre forces. SAE paper 900210, 1990.

18 Zhou, J., Wong, J. Y., and Sharp, R. S. A multi-spoke, three plane tyre model for simulation of transient behaviour. Veh. Syst. Dynamics, 1999, 31, 35-45.

19 Sharp, R. S. and El-Nashar, M. A. A generally applicable digital computer based mathematical model for the generation of shear forces by pneumatic tyres. Veh. Syst. Dynamics, 1986, 15, 187-209.

20 Mavros, G., Rahnejat, H., and King, P. D. Investigation of steady-state tyre force and moment generation under combined longitudinal and lateral slip conditions. 18th LAVSD Symposium on Dynamics of
Vehicles on Roads and Tracks, Atsugi Kanagawa, Japan, August 2003.

21 Yu, Z.-X., Tan, H.-F., Du, X.-W., and Sun-Li. A simple analysis method for contact deformation of rolling tire. Veh. Syst. Dynamics, 2001, 36(6), 435-443.

22 Braghin, F., Cheli, F., and Resta, F. Friction law identification for rubber compounds on rough surfaces at medium sliding speeds. 3rd AIMETA International Tribology Conference, Salerno, Italy, 18-20 September 2002.

23 Karnopp, D. Computer simulation of stick-slip friction in mechanical dynamic systems. Trans. ASME, J. Dynamic Syst. Measmt. and Control, 1985, 107, $100-103$.

\section{APPENDIX}

\section{Notation}

b

C

$D_{\text {inter }}$

$D_{x x}$

$D_{y x}$

$\boldsymbol{f}, f_{x}, f_{y}$

$F_{\text {vertical }}$

$F_{z}$

$g$

$K_{\text {inter }}$

$K_{x x}$

$K_{y x}$

$l=2 \cdot a$

$n$

$R$

patch

$R_{\mathrm{d}} \quad$ radius of the free rolling vertically loaded tyre

$u \quad$ velocity of the tread element on the road (oxy) plane

$u_{x} \quad$ longitudinal component of $u$

$u_{y} \quad$ lateral component of $u$

$V_{\mathrm{d}}=V_{\mathrm{r}} \quad$ longitudinal velocity of travel of the tread element in the moving frame of reference

$V_{\mathrm{s} x} \quad$ longitudinal sliding velocity of the tread

$V_{\mathrm{sy}} \quad$ lateral sliding velocity of the tread

$\mu \quad$ coefficient of static friction

$\mu_{\mathrm{k}} \quad$ coefficient of kinetic friction

$\omega \quad$ rotational velocity of the wheel 